Robert Kowarsch*, Jürgen Janzen, Hyunjun Cho, Hyuck Choo und Christian Rembe

\title{
Scanning confocal vibrometer microscope for vibration analysis of energy-harvesting MEMS in wearables
}

\author{
Scannendes konfokales Vibrometermikroskop für die Schwingungsanalyse von Energy-Harvesting- \\ Microsystemen für Wearables
}

DOI 10.1515/teme-2017-0042

\begin{abstract}
We present a scanning confocal laser-Doppler vibrometer microscope for sensitive, contactless measurement of microelectromechanical systems (MEMS). This systems enables the dynamic analysis up to $3.2 \mathrm{MHz}$ with a lateral resolution of few micrometers. We show measurements on developed MEMS for vocal-energy harvesting in wearables and medical implants. For efficient harvesting a cantilever beam with a serpentine form was designed with a fundamental resonance at $200 \mathrm{~Hz}$. We verified the simulated mode shapes with our vibration measurements. The observed deviations in resonance frequencies between simulation and measurement are due to modelling and manufacturing dissimilarities.
\end{abstract}

Keywords: Laser-Doppler vibrometry, interferometric measurement microscope, energy harvesting, MEMS, microsystems, vibration analysis.

Zusammenfassung: Wir stellen ein scannendes konfokales Laser-Doppler-Vibrometermikroskop für die empfindliche und berührungslose Messung von Mikrosystemen vor. Unser System erlaubt die dynamische Ana-

\footnotetext{
*Corresponding author: Robert Kowarsch: Clausthal University of Technology, Institute of Electrical Information Technology, Department of Applied Metrology, Leibnizstraße 28, 38678 Clausthal-Zellerfeld, e-mail: kowarsch@iei.tu-clausthal.de Jürgen Janzen, Christian Rembe: Clausthal University of Technology, Institute of Electrical Information Technology, Department of Applied Metrology, Leibnizstraße 28, 38678 Clausthal-Zellerfeld, e-mail: rembe@iei.tu-clausthal.de Hyunjun Cho, Hyuck Choo: California Institute of Technology, Department of Electrical Engineering, B113 Moore Laboratory, MC 136-93, Pasadena, CA 91125, e-mail: hccho@caltech.edu, hcchoo@caltech.edu
}

lyse der Bauteile bis zu einer Frequenz von 3.2 MHz bei einer lateralen Auflösung von wenigen Mikrometern. Wir zeigen Messungen an entwickelten Mikrosystemen zum Energie-Harvesting aus Stimmanregung für Wearables und medizinische Implantate. Für das effiziente Harvesting wurde ein serpentinenförmiger Cantilever entwickelt, der eine Resonanz bei $200 \mathrm{~Hz}$ besitzt. Wir verifizierten die simulierten Schwingformen mit unseren Schwingungsmessungen. Abweichungen der gemessenen Resonanzfrequenzen zwischen der Simulation und den Messungen sind auf Unterschiede zwischen Modell und gefertigtem Bauteil zurückzuführen.

Schlüsselwörter: Laser-Doppler-Vibrometrie, interferometrisches Messmikrsokop, Energy-Harvesting, MEMS, Mikrosysteme, Vibrationsanalyse.

\section{Introduction}

Heterodyne laser-Doppler vibrometers leveraged to an indispensable part in the development and manufacturing of microelectromechanical systems (MEMS) [8]. These instruments satisfy the need for the analysis of dynamic response, providing a nondestructive and contactless method with the necessary high spatial resolution and motion-amplitude resolution with a large measurement bandwidth. A laser-Doppler vibrometer was first incorporated by Burdess for MEMS testing [1]. Today commercially-available microscopic measurement systems are state-of-the-art and even cover the requirement of $\mathrm{GHz}$ measurement [9] or sensitive 3D analysis [11].

During the last years the integration of electrical sensors and systems in and at the human body (so called wearables) has steadily emerged. These systems can be either for medical, military or entertainment purposes. 
Most systems are currently powered by a battery packs, which have to be recharged periodically. For completely energy-self-sufficient systems the renewable power sources can be heat, solar energy, radio-frequency electromagnetic radiation and vibrations. As vibration is omnipresent in and at the human body, this approaches for energy-harvesting received wide attention. In contrast, energy harvesting for wearables from solar and heat energy is dependent on the ambience of the person and, therefore, is not able to provide a constant power supply. Our energy-harvesting MEMS exploit the human vocal energy, whose dominant frequency is in the range of 100 to $300 \mathrm{~Hz}[3,4]$, which can be applied for wearables or medical implants. Further, these energy harvesters are also suitable for energy self-sufficient sensor nodes exploiting vibrations of typical environmental sources, which typically have a low level of vibration below $1 \mathrm{~g}$ at frequencies below $200 \mathrm{~Hz}$ [12].

In this paper, we present our scanning confocal laserDoppler vibrometer-microscope providing the requirements for dynamic MEMS analysis. We further show vibration measurements on MEMS of serpentine form for vocal-energy harvesting. From our scanning measurement, we reconstructed the out-of-plane mode shapes and compared these vibration measurement results with our COMSOL simulations.

\section{Material and methods}

\subsection{Energy-harvesting MEMS}

To exploit vocal vibrations for energy harvesting, it is required to build MEMS which resonates at the dominant frequency of vocal vibrations (100 to $300 \mathrm{~Hz}$ ) [3]. For the piezoelectric material, lead zirconate titanate (PZT) sheet with bimorph structure (totally $510 \mu \mathrm{m}$ thick) was chosen due to its high piezoelectric coupling coefficient $[2,7]$.

Under free vibration (with a single degree of freedom) a piezoelectric beam, modelled as uniform cantilever, generally follows the differential equation [13]

$$
E I \frac{\mathrm{d}^{4} u_{z}(x)}{\mathrm{d} x^{4}}=\omega^{2} m u_{z}(x)
$$

with the modulus of rigidity $E$, and the moment of inertia $I$ of the rectangular beam cross-section $\left(I=b d^{3} / 12\right)$ with the thickness $d$ and width $b$. $\omega$ is the angular natural frequency, $m$ is the mass per unit length, and $u_{z}(x)$ is the out-of-plane displacement at the distance $\mathrm{x}$ from the clamped end.

For the mode shape for a rectangular beam of length 1 , it follows for the undamped natural frequencies ( $r$ th bending mode) from the differential Equation 1 (shortcircuit condition) in dependency of the beam dimensions

$$
f_{r} \propto \sqrt{\frac{b d^{3}}{l^{4}}} .
$$

Thus, a thick piezoelectric beam is usually inappropriate for the use of low frequency applications like 100$300 \mathrm{~Hz}$ because the thicker beam makes the beam stiffer (see Equation 2), so that it results in higher resonance frequency $f_{r}$ of the beam. To overcome this problem, we chose a serpentine-shaped beam which has longer effecttive beam length compared to a uniform rectangular beam within a given area.

From the solution of Equation 1, the vibration nodes of the mode shapes can be calculated [13] which are not equidistant especially for lower frequencies. However, the node spacing at the $r$ th bending mode can be estimated (with the assumption of fixed end instead of clamping) to

$$
\Delta x_{r} \approx l /(r-0.5)
$$

For the measurement of the vibrating cantilever the deflection modes have to be resolved by the scanning measurement method. According to the Nyquist criterion the distance between two adjacent measurement points is to be chosen smaller than half the node spacing.

However, the Euler-Bernoulli beam assumption (Equation 1) oversimplifies the dynamics of a beam, especially for the serpentine shape. Therefore, using COMSOL Multiphysics simulations, we compared the frequency responses of the serpentine beam with that of the rectangular beam. We set the dimension to be $12.7 \times 13.5 \mathrm{~mm}^{2}$. As shown in Figure 1, the fundamental frequency of the serpentine beam is $200.6 \mathrm{~Hz}$, whereas for the same are a rectangular beam shows a fundamental frequency at $1363 \mathrm{~Hz}$. We also simulated the displacement at the tip of the cantilever as the frequency varied from 0 to $1000 \mathrm{~Hz}$, given that the applied force was $0.4 \mathrm{~g}$ (Figure 1). The largest displacement occurs at the fundamental frequency and other modes follow at 346.5, 746.0, and $961.1 \mathrm{~Hz}$.

For the fabrication of the beam, we applied the lasermicromachining process (ND:YAG-UV 355). The brittle property of PZT was the major huddle to shape the serpentine structure. To manage the crack which usually 
occurs at the corner, ultra-short pulse laser like picosecond or femtosecond laser has been used [5]. This ultra-short pulse laser process enables high power laser cutting process with minimized thermal effects. With the use of picosecond laser (pulse width: 8 ps), any crack has not been found on the fabricated structure.

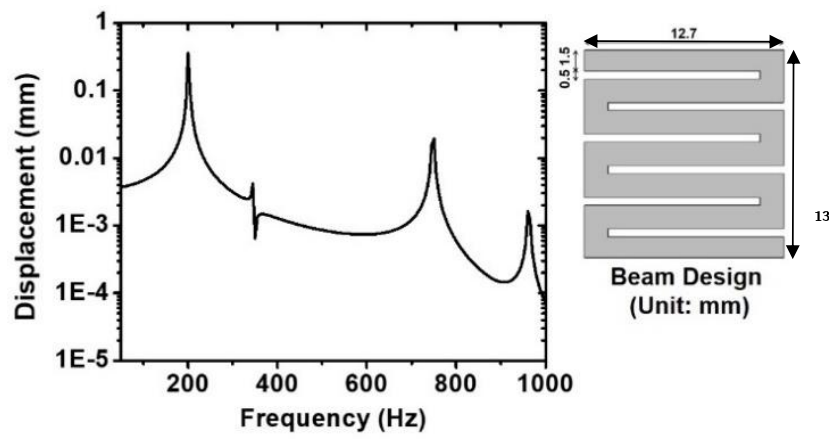

Figure 1: COMSOL Multiphysics simulations: Serpentine beam design and the calculation of the beam displacement at the tip over frequency.

\subsection{Heterodyne laser-Doppler vibrometry}

For the contactless vibration measurement of the MEMS, we employ the technique of heterodyne laser-Doppler vibrometry in this paper. Thus the main principles of laser-Doppler effect [6] and a heterodyne-vibrometer setup are summarized herein. For slow vibrations (compared to the speed of light) a displacement $z(t)$ of the measurement object in the direction of the optical axis (out-of-plane) changes the optical path length of $2 z(t)$. Thus, the backscattered laser radiation gets phasemodulated at moving objects. The electric field of coaxially-backscattered radiation can be expresses as

$$
\begin{aligned}
E_{m}(t) & =\widehat{E}_{m} \cos \left(\omega_{m} t-\varphi_{m}(t)\right) \\
& =\widehat{E}_{m} \cos \left(\omega_{m} t-2 k_{m} z(t)+\varphi_{m, 0}\right)
\end{aligned}
$$

with the constant phase $\varphi_{0}$ due to propagation, the angular frequency $\omega=k c$, the wavenumber $k$ and the velocity of light $c$. For the reconstruction of the vibration the measurement point a broadband demodulation of the phase $\varphi(t)$ of the electric field is required. A common technique to access the phase of rapidly oscillating electromagnetic fields (at $\omega=4.7 \times 10^{14} 1 / \mathrm{s}$ ) provides the interferometry. Therefore, the modulated electric field is coherently interfered with an unmodulated electric field of the form

$$
E_{r}(t)=\widehat{E}_{r} \cos \left(\omega_{r} t-\varphi_{r, 0}\right)
$$

on a photodiode, which acts as a quadratic mixer. For the realization common interferometer setups, e.g. MachZehnder setup, can be applied [10]. With its characteristic sensitivity $S$, the photodiode delivers an output current

$$
i_{p h}(t)=S\left|E_{r}(t)+E_{m}(t)\right|^{2} .
$$

Since the sum-frequency is also beyond the bandwidth of common photodiodes, the photodiode current is (with Equations 4 and 5)

$$
\boldsymbol{i}_{p h}(t)=\frac{s}{2}\left(\widehat{E}_{r}^{2}+\widehat{E}_{m}^{2}+\widehat{E}_{r} \widehat{E}_{m} \cos \left[\left(\omega_{m}-\omega_{r}\right) t-\varphi_{m}(t)-\right.\right.
$$

with the constant phase offset $\varphi_{0}=\varphi_{m, 0}-\varphi_{r, 0}$.

It is advantageous to incorporate different angular frequencies $\left(\omega_{m} \neq \omega_{r}\right)$. Thus, a frequency-modulated (FM) photodiode current with the heterodyne carrier frequency $\omega_{c}=\omega_{m}-\omega_{r}$ is generated and for the demodulation common FM demodulation methods can be applied.

In summary, the frequency shift (Doppler shift) is proportional to the out-of-plane velocity $v_{z}(t)=$ $\mathrm{d} u_{z}(t) / \mathrm{d} t$ of the moving object at the measurement point

$$
f_{D}(t)=\frac{\mathrm{d}}{\mathrm{d} t} \varphi_{m}(t)=2 k_{m} \frac{\mathrm{d}}{\mathrm{d} t} u_{z}(t)=2 k_{m} v_{z}(t)
$$

\subsection{Vibrometer microscope}

Our optical setup consists of a scanning microscope, which provides a coupling with laser vibrometers in the spectral range of 530 to $650 \mathrm{~nm}$ with a variable beam diameter. For the presented measurements we used a heterodyne laser-Doppler vibrometer OFV-353S from Polytec, which uses the HeNe wavelength of $632.8 \mathrm{~nm}$. The scanning of the measurement object is done by the linear-motor-driven $x y$ stage KT230-EDLM from Steinmeyer Mechatronics with a position uncertainty of $\pm 2 \mu \mathrm{m}$ and a full range of $100 \mathrm{~mm} \times 100 \mathrm{~mm}$. The stage is controlled via a LabVIEW program.

For determining the measurement points and geometry alignment, a camera observers the measurement object through a dichroitic mirror. The suppression of the dichroitic mirror at the laser wavelength has been chosen to sufficiently image the measurement spot on the object. Combined with a shorter focal length of the tube lens the camera has a field-of-view of $280 \mu \mathrm{m} \times 450 \mu \mathrm{m}$ to $2.8 \mathrm{~mm} \times 4.5 \mathrm{~mm}$. A broad-band LED with a large 
emission angle homogenously illuminates the measurement volume according to the Köhler illumination.

We combined the bright-field illumination and the imaging path with the same optics. The tube lens is therefore both used for imaging into the camera and for imaging the LED to the exit pupil of the microscope objective. This leads to a compact design of the upright microscope with a height (sample to top) of $350 \mathrm{~mm}$ and width of $230 \mathrm{~mm}$.

\subsection{Confocalization}

In previous works, we showed that for coherent detection in vibrometers the reference beam and the limited size of the photodiode are forming a quasi-pinhole [11]. This results in a confocal detection characteristic of the vibrometer microscope (not for the camera). For potential confinement, a pinhole is introduced in the Keplerian telescope after the vibrometer. However, a Gaussian intensity distribution in the exit pupil of the microscope objective is beneficial to achieve a Gaussian point-spread function (PSF) on the mea-surement object [10]. Therefore, the beam diameter is diminished to be smaller than the exit-pupil diameter of the microscope objective. This is superior to an Airy PSF, which shows side maxima. Those side maxima may interfere with the signal from the central maximum causing motion amplitude errors due to multi-wave mixing. However, with our method the $N A$ of the microscope objective is not exploited, so that the effective $N A$ is smaller. This causes larger PSFs than

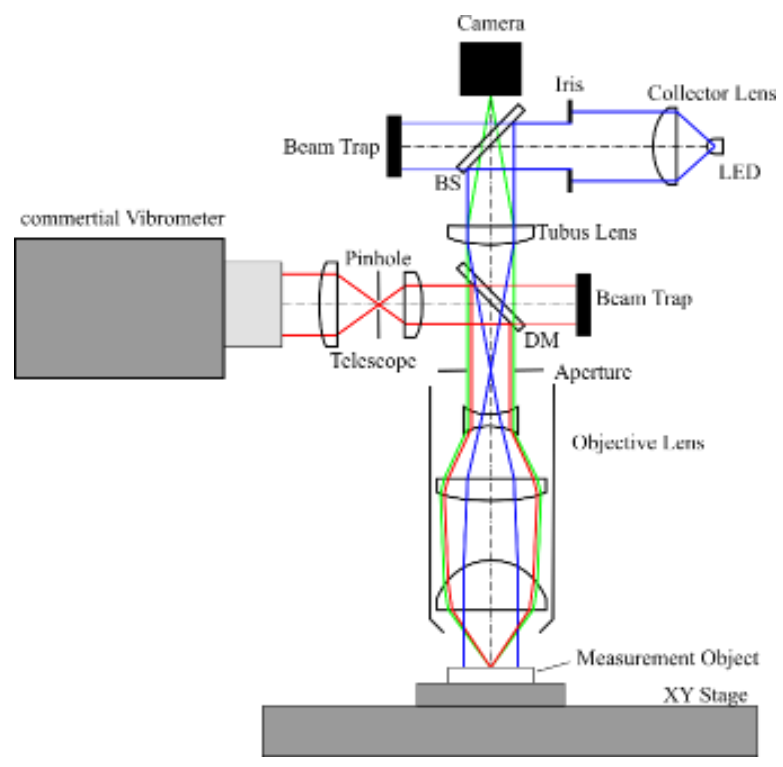

theoretically achievable with the objective. With a flexible interchange of different microscope objectives from Mitutoyo, we are able to achieve theoretic PSF diameters in the range of $1.4 \ldots 5.5 \mu \mathrm{m}$. The nominal spot size of the OFV-353S is $15 \mu \mathrm{m}$. In addition, the utilized microscope objectives allow comfortable adjustment of the measurement object due to a large working distance.

\subsection{Signal processing}

With the incorporated Polytec OFV-2500-2 decoder we are able to measure out-of-plane vibrations on MEMS up to $3.2 \mathrm{MHz}$, which is sufficient for the frequency range of the developed energy-harvesters. This system bandwidth even enables testing of MEMS up to several MHz.

For the comparison to the COMSOL simulations, we reconstructed the operating deflection shapes (ODS) from the sequential measurements. The necessary phase reference is therefore the excitation signal, which was chosen as a periodic chirp from 50 to $4000 \mathrm{~Hz}$. For the dynamic analysis, the excitation principle of the serpentine beam was reversed by applying a harmonic voltage signal and observing the vibration. The exciting voltage amplitude was $5 \mathrm{~V}$ peak-to-peak. Additionally, the period of the periodic chirp was adapted to the FFT window length, which enables a high reduction of leakage and fast measurement. The FFT was set to 12800 FFT lines at sampling frequency of $10 \mathrm{kHz}$ providing a resolution bandwidth of $0.78 \mathrm{~Hz}$ (measurement duration

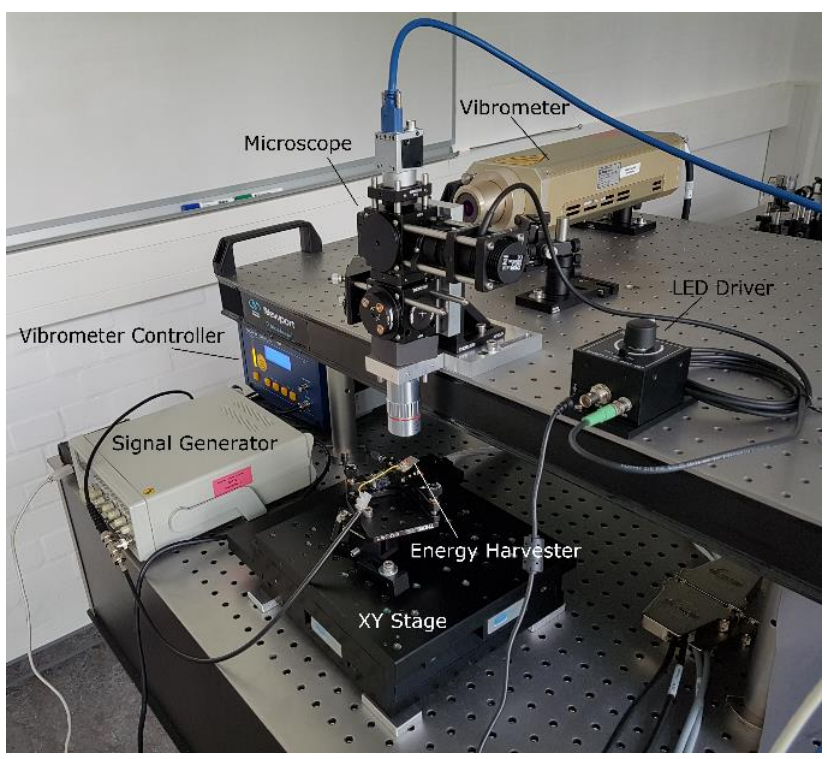

Figure 2: Schematic of the of the scanning confocal vibrometer microscope (left). A dichroitic mirror (DM) superimposes the vibrometer beam with the microscope optics. The Köhler illumination is provided by a LED. The photograph (right) shows the whole measurement setup. 
of $1.28 \mathrm{~s})$. For the final visualization and analysis of the measured ODS, we synchronously display the sequential measurements in MATLAB. No spatial averaging of nearby points was applied.

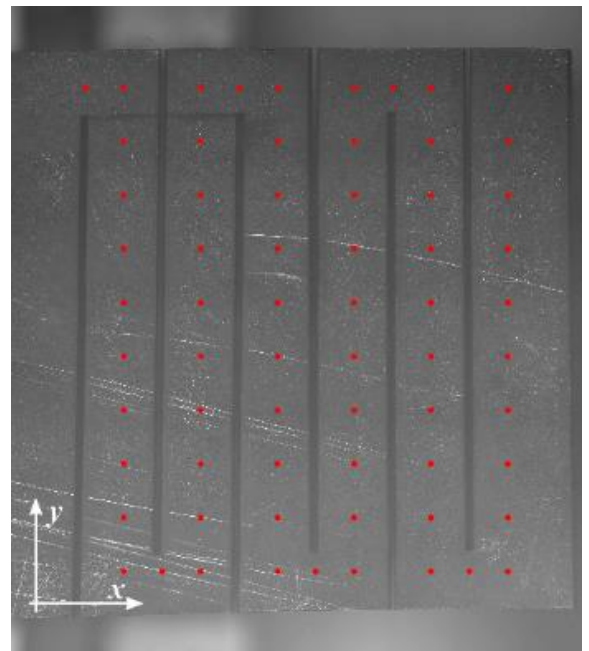

Figure 3: Stiched image of the serpentine beam with the measurement points (red). Each single image was acquired with the incorporated camera and the $5 \times$ microscope objective.

\section{Results and discussion}

For the evaluation of the energy-harvester, we chose a $5 \times$ microscope objective to achieve a field-of-view of $2.8 \mathrm{~mm} \times 4.5 \mathrm{~mm}$. For the geometry alignment, the partial images of the energy-harvester were stitched together allowing a determination of the measurement points. We chose 66 equidistant measurement points linearly distributed along the serpentine beam (see Figure 3) on the serpentine beam with an average spacing of $1.22 \mathrm{~mm}$ in $y$ and $0.9 \mathrm{~mm}$ in $x$. According to the assumption (Equation 3), this enables the resolution of maximum the third bending mode shape in $x$ direction and even higher modes in $y$ direction. Additionally, for each measurement point a complex averaging of 25 measurements was conducted to reduce noise.

With our vibrometer microscope we sequentially measured the electromechanical transfer function $H_{1}[8]$ at each predefined point of the serpentine beam. The outof-plane velocity measurement at an exemplary measurement point at the tip of the beam (see Figure 4) shows several characteristic resonances. Since these resonance peaks are widely spaced, we assume that the measured ODS at the resonance peaks are approximately the mode shapes of the serpentine beam and can, thus, be compared to the mode shapes of the simulation. In Table 1 the first four dominant out-of-plane mode shapes are compared between simulation and measurement. Further, the corresponding mode shapes of the simulated rectangular beam are shown. Generally, the serpentine beam with the chosen size and cross section is design to have its first bending mode in the range of dominant vocal frequencies (100 to $300 \mathrm{~Hz}$ ). Further resonances below the maximum vocal frequency $(3000 \mathrm{~Hz})$ also enable harvesting. However, the main vocal energy is below $300 \mathrm{~Hz}[3]$.

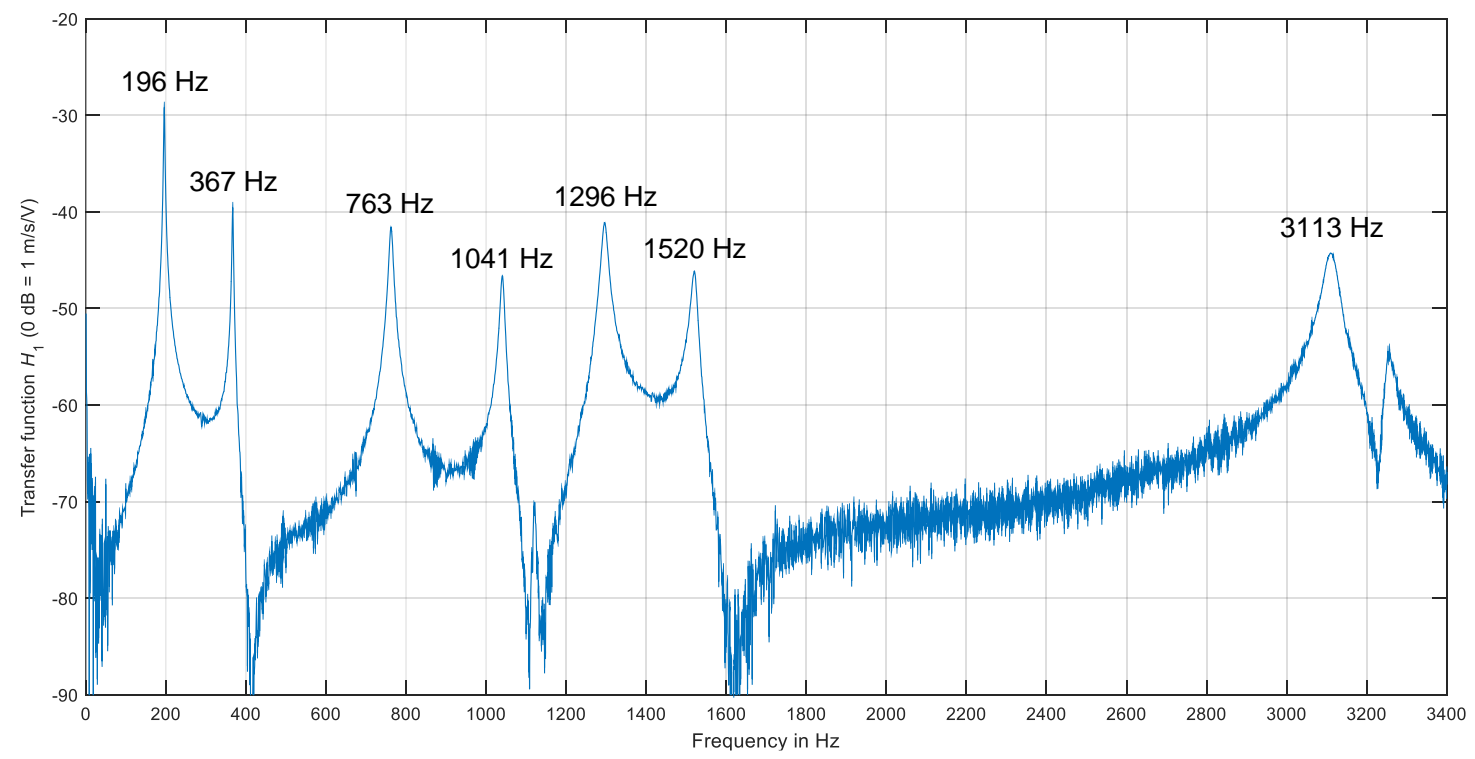

Figure 4: Electromechanical transfer function $H_{1}$ at the tip of the serpentine beam measured with our vibrometer microscope. The curve shows the spectral out-of-plane motion-velocity response per Volt (harmonic excitation amplitude) normalized to a factor of $1 \mathrm{~m} / \mathrm{s} / \mathrm{V}$. This spectrum shows several resonance peaks (see also Table 1) which can be assigned to modes shapes of this MEMS. 
Table 1: Comparison of out-of-plane mode shapes and resonance frequencies of the serpentine beam between simulation and measurement. The mode shapes are qualitatively presented in the state of largest displacement amplitude (largest displacement shown red) omitting the first digit, which is immobile due to clamping.

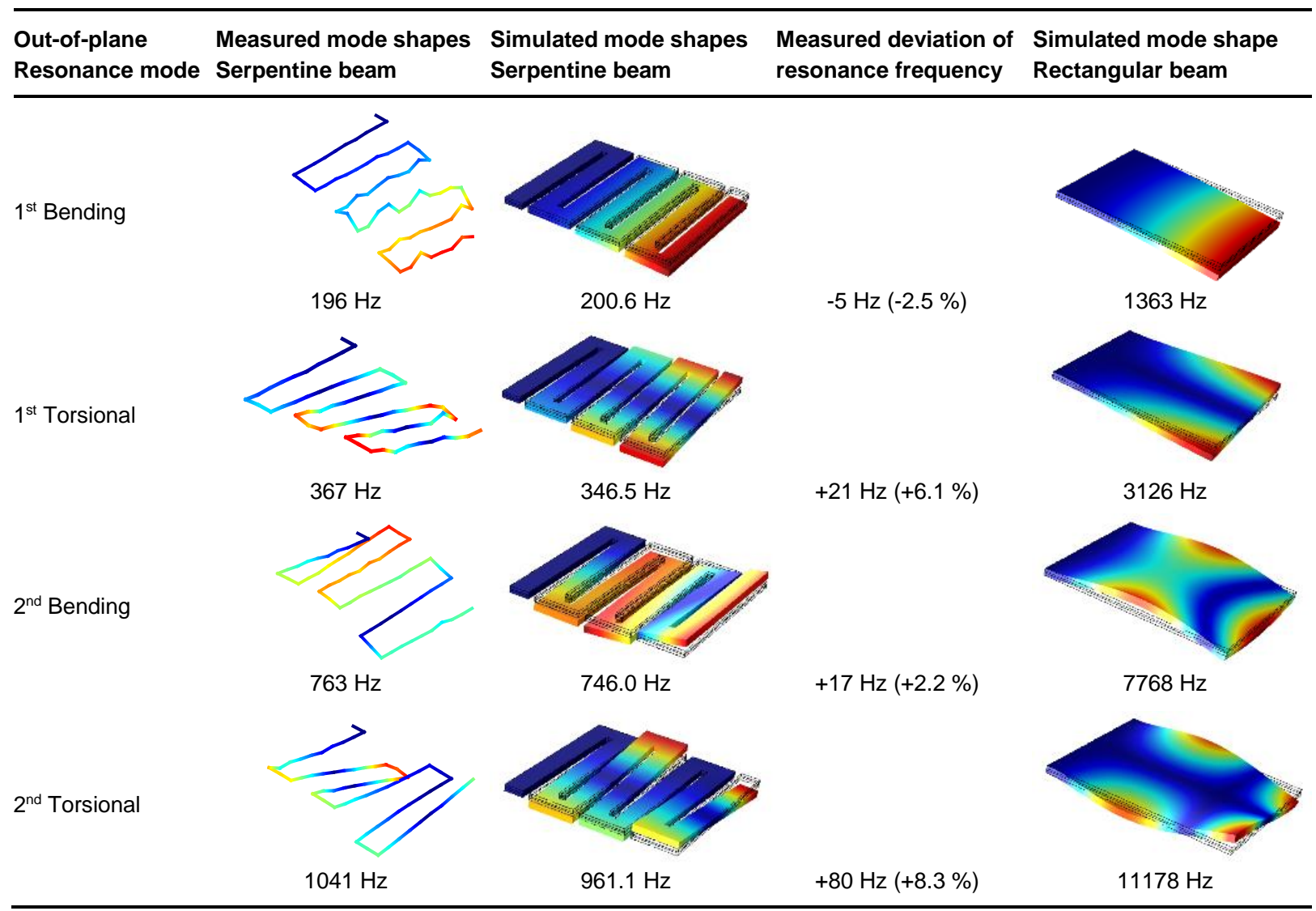

Similar to the simulations, the first four (bending and torsional) mode shapes show a global behaviour comparable to a rectangular beam. Due to the slits, the frequency of the fundamental bending resonance is reduced of more than factor of 6.8 with the serpentine form. Further, the second bending resonance for the serpentine beam occurs at a multiple of 3.9 of the fundamental frequency, whereas for a rectangular beam it occurs at a multiple of 6.3. Thus, also the spacing of the orders of resonance are reduced beneficially for vocal-energy harvesting.

The measured mode shapes of the serpentine beam verify the theoretical behaviour in the simulation. The differences in resonance frequencies are mainly due to parameter deviations, imperfections of the material and deviations of the geometry due to material processing. Especially, the larger digit at the tip of the beam (see Figure 3) can be responsible for the detuning of the resonances compared to the simulated ideal shape.
Further resonances can be observed above $1050 \mathrm{~Hz}$ (see Figure 5), which were not investigated in the simulation. These resonances show a local behaviour, which means that the several segments move out-ofphase (flapping).

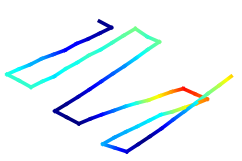

$1296 \mathrm{~Hz}$

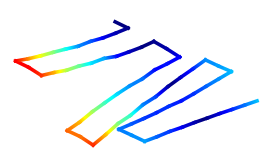

$1520 \mathrm{~Hz}$

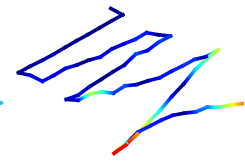

$3113 \mathrm{~Hz}$
Figure 5: Flapping ODS of the serpentine beam at dominant resonance frequencies $>1100 \mathrm{~Hz}$ of the transfer function $H_{1}$ (see Figure 4). 


\section{Conclusion and outlook}

For the vibration analysis, we setup a confocal laserDoppler vibrometer microscope, which enables vibration measurement on MEMS with microscopic resultion down to a feature size of few microns. With the employed heterodyne vibrometer and decoder with a measurement bandwidth of $3.2 \mathrm{MHz}$, we analysed and reconstructed the operating deflection shapes of the developed serpentine beam for vocal-energy harvesting at the dominant vocal frequencies (100 to $300 \mathrm{~Hz}$ ) for wearables or medical electronic implants. The chosen serpentine shape allows to reduce the fundamental resonance frequency within the same area of $12.7 \times 13.5 \mathrm{~mm}^{2}$ up to a factor of 6.8 compared to a rectangular beam.

The simulations results with COMSOL predict a fundamental resonance frequency for the serpentine beam of $200.6 \mathrm{~Hz}$. The vibration measurements with our vibrometer microscope show the first bending resonance at $196 \mathrm{~Hz}$, thus, only with a difference of $-4 \mathrm{~Hz}$. Also higher mode shapes and resonance frequencies are consistent between simulation and measurement and the frequency deviations are less than $8 \%$.

In the future, the measurement results will be used to update the model for the COMSOL simulation. This will help both to improve the results and accelerate the computer-aided design process to achieve efficient energy-harvesters. We will further improve our vibrometer microscope to improve the adjustment and geometry alignment for a better reconstruction of the operating deflection shapes.

Acknowledgment: The project on energy harvesters has been funded by the Caltech CI2 program, Powell Foundation, and Heritage Foundation.

\section{References}

1. J. S. Burdess, A. J. Harris, D. Wood, R. J. Pitcher, and D. Glennie. A system for the dynamic characterization of microstructures. Journal of Microelectromechical Systems, 6(4): 322-328, 1997.

2. A. Chaipanich. Dielectric and piezoelectric properties of PZT-cement composites. Current Applied Physics, 7(5):537539, 2007.

3. H. Cho, A. Balakrishna, Y. Ma, J. O. Lee, and H. Choo. Efficient power generation from vocal folds vibrations for medical electronic implants. IEEE MEMS 2016, 363-366, 2016.
4. H. Cho, K. Noh, T. Ishikawa, D. Yang, and E. SánchezSinencio, Powering portable electronics using vocal fold vibrations. IEEE MEMS 2017, 217-220, 2017.

5. J.-P. Desbiens, and P. Masson. ArF ecximer laser micromachining of Pyrex, $\mathrm{SiC}$ and PZT for rapid prototyping of MEMS components. Sensors and Actuators A, 136, 554563, 2007.

6. C. Doppler. Ueber das farbige Licht der Doppelsterne und einiger anderer Gestirne des Himmels. Versuch einer das Bradley'sche Aberrations-Theorem als integrirenden Theil in sich schliessenden allgemeineren Theorie. Borrosch \& André, 1842.

7. C. G. Hindrichsen, R. Lou-Møller, K. Hansen, and E. V. Thomsen. Advantages of PZT thick film for MEMS sensors. Sensors and Actuators A: Physical, 163(1): 9-14, 2010

8. W. Osten. Optical inspection of microsystems: Chapter 9: Measuring MEMS in Motion by Laser Doppler Vibrometry. CRC/Taylor \& Francis Boca Raton, FL, 1st ed., 2007.

9. C. Rembe, S. Boedecker, A. Dräbenstedt, F. Pudewills, and G. Siegmund. Heterodyne laser-Doppler vibrometer with a slow-shear-mode Bragg cell for vibration measurements up to $1.2 \mathrm{GHz}$. Proceedings of 8th Int. Conference on Vibration Measurements by Laser Techniques - AIVELA, ed. E. P. Tomasini, 70980, June 2008.

10. C. Rembe, and A. Dräbenstedt. Laser-scanning confocal vibrometer microscope. Theory and experiments. Rev. Sci. Instrum. 77(8): 83702, 2006.

11. C. Rembe, R. Kowarsch, W. Ochs, A. Dräbenstedt, M. Giesen, and M. Winter. Optical three-dimensional vibrometer microscope with picometer-resolution in $\mathrm{x}, \mathrm{y}$, and $\mathrm{z}$. Opt. Eng. 53(3): 34108, 2014.

12. S. Roundy, P. K. Wright, and J. Rabaey. A study of low level vibrations as a power source for wireless sensor nodes. Computer Communications, 26(11): 1131-1144, 2003.

13. W. T. Thomson, and M. D. Dahleh. Theory of vibration with applications. Prentice Hall Upper Saddle River, NJ, 5th ed., 1998. 